\title{
Dificultades de desarrollo local en los municipios y su vínculo con la Educación Superior.
}

\section{Local- development difficulties in the municipalities and his link with the education Superior.}

MSc. Dagmar Santa Cruz Pérez ${ }^{1}$, Dra.C Victoria Ojalvo Mitrany ${ }^{2}$, Msc. Yulima D. Valdés Bencomo $^{3} \&$ Dr.C. Efraín Velasteguí López.

Recibido: 10-11-2018 / Revisado: 17-02-2019 /Aceptado: 25-02-2019/ Publicado: 05-04-2019

\begin{abstract}
.
DOI: https://doi.org/10.33262/cienciadigital.v9i2.355

The study addresses a dissertation about the obstacles and difficulties encountered from the research carried out by Dr. C. Jorge Núñez Jover (2012-2013) "University management program of knowledge and innovation for development," and an analysis of the author of this study based on the above and its impact at present in the community context, which is added as the main factor of the role of governments as top decision makers in the search for possible solutions from the policy process of institutional and communication management. The transformations that the Cuban State proposes at present demand a position of change of traditional schemes that in their historical moment fulfilled their function, but now they demand of a redesign and organic and holistic thought according to the demands that are imposed in the reality historical and sociocultural of the Cuban people.

These demands demand the notable presence of knowledge management through the predominant role of Higher Education as an agent of local development based on the link University-society-company and University-local government. Finally, the role of innovation and the policy of institutional communication in local governments are key axes in the development and development of the dynamics of local development in the municipalities, where understanding it becomes a challenge to achieve in the central objective of the Higher education as agent of local development.
\end{abstract}

Keywords: University Management of Knowledge, Innovation, Local Development.

\footnotetext{
${ }^{1}$ Universidad de la Habana, Rectorado, La Habana, Cuba, dstacruz@ rect.uh.cu

${ }^{2}$ Universidad de la Habana, CEPES, La Habana, Cuba, victoria@cepes.uh.cu

${ }^{3}$ Universidad de la Habana, Facultad de Turismo, La Habana, Cuba, ybencomo@ftur.uh.cu

${ }^{3}$ Ciencia Digital, Ambato, Ecuador luisefrainvelastegui@cienciadigital.org
} 


\section{Resumen.}

El estudio aborda una disertación acerca de los obstáculos y dificultades encontradas a partir de la investigación realizada por el Dr. C. Jorge Núñez Jover (2012-2013) "Programa de gestión universitaria del conocimiento y la innovación para el desarrollo, "y un análisis del autor de este estudio a partir de lo expuesto y su impacto en la actualidad en el contexto comunitario, donde se añade como factor principal el papel de los gobernantes como máximos decisores en la búsqueda de posibles soluciones desde el proceso de política de gestión institucional y comunicacional. Las transformaciones que se propone el Estado cubano en la actualidad demandan una postura de cambio de esquemas tradicionales que en su momento histórico cumplieron su función, pero ahora exigen de un rediseño y pensamiento orgánico y holístico de acuerdo a las demandas que se imponen en la realidad histórica y sociocultural del pueblo cubano.

Estas demandas exigen la presencia notable de la gestión del conocimiento a través del papel predominante de la Educación Superior como agente de desarrollo local a partir del vínculo Universidad - sociedad-empresa y Universidad- gobierno local. Por último, el papel de la innovación y la política de comunicación institucional en los gobiernos locales son ejes claves en el desenvolvimiento y desarrollo de la dinámica del desarrollo local en los Municipios, donde entenderlo se convierte en un reto a alcanzar en el objetivo central de la Educación Superior como agente de desarrollo local.

Palabras claves: Gestión Universitaria del Conocimiento, Innovación, Desarrollo Local.

\section{Introducción.}

Hoy más que nunca la realidad cubana está urgida de los cambios que se proponen en los lineamientos del VI Congreso que acompañan al desarrollo local, esto no es magia que toca algo y lo cambia, de lo que se trata es de construir a partir de las conceptualizaciones en torno al desarrollo local. Al adentrarse más en la identificación de los obstáculos que frenan el desarrollo local en Cuba, se observa que, en una investigación realizada por un grupo multidisciplinario encabezado por el Dr. C. Jorge Núñez Jover (2012-2013) "Programa de gestión universitaria del conocimiento y la innovación para el desarrollo," fueron detectados los principales obstáculos; ellos son:

1. "Centralización y falta de autonomía en los municipios.

2. Desarticulación y sectorialismo en el trabajo de las instituciones y proyectos.

3. Obstáculos de naturaleza jurídica. Limitaciones en la participación social en los procesos de toma de decisiones.

4. Necesidades de generación de capacidades para el desarrollo local.

5. Coexistencia de pluralidad de nociones de desarrollo local. 
6. Aprovechamiento limitado de los conocimientos, tecnologías e innovaciones locales.

7. Limitaciones en el acceso de información relevante para el ejercicio del desarrollo local.

8. Limitaciones de recursos relacionados con la infraestructura productiva y las condiciones de trabajo, materiales con que cuentan los actores locales para el ejercicio de sus funciones.

9. El insuficiente aprovechamiento o la ausencia de claridad en el rol de la cooperación internacional.

Otra importante fuente en la detección de problemas son los relativos a la comunicación encontrados por el autor de la investigación desde la dinámica de trabajo con las alianzas establecidas con el gobierno local como: barreras de comunicación y prácticas comunicativas ineficientes traducidas en errores, desaciertos, una postura y toma de decisiones inadecuadas, dificultades para clarificar bien las tareas y los mensajes y poder cumplir con eficiencia y calidad las orientaciones e informaciones tributadas por las diferentes comunidades implicadas a partir de sus necesidades sentidas y conscientemente reconocidas.

Esto trajo como resultado la pérdida de oportunidades observadas en la implementación de importantes proyectos, como los derivados de documentos rectores, nacionales e internacionales en convenios realizados por el gobierno local. Por lo tanto, la dinámica del gobierno local exige el análisis de la situación de su funcionamiento desde su posicionamiento como órgano de base gubernamental con la debida articulación de todos los actores implicados.

El hecho de que las transformaciones que hoy se propone el país entrañen cambios sustanciales, justifica la necesidad de desaprender conceptos y esquemas de trabajo tradicionales, para construir una cosmovisión diferente acerca de cómo impulsar los procesos de desarrollo local.

Esta meta, si bien de fácil descripción, expone una concreción compleja, que encontrará sus principales obstáculos en la subjetividad de quienes no han percibido que, aunque el objetivo sigue siendo el mismo: alcanzar un desarrollo sostenible y sustentable, se impone la asimilación de variantes innovadoras para alcanzarlo. En este escenario, el desarrollo de los municipios cubanos y los procesos de innovación asociados a estos pasan a ser centro de atención de los decisores al más alto nivel. Sin embargo, no basta que estos lo entiendan, e incluso tomen medidas al respecto. Núñez Jover (2013)

Se precisa que los gobernantes y demás actores de la escala local comprendan la tamaña tarea de ser protagonistas de una concepción innovadora de gestar el progreso socio-económico de su territorio, donde la gestión municipal depende, de que los decisores perciban el desarrollo local y la comunicación como dos procesos dinámicos de cambios estructurales generados por los actores sociales de un territorio. 
Recientemente, la ley le ha concedido a los municipios un mayor grado de autonomía, que les permite consolidar los diferentes procesos de gestión desde la institución, con la inclusión de los actores sociales implicados y la comunidad. Para que la autonomía sea efectiva, es preciso un mayor grado de compromiso y responsabilidad en la toma de decisiones, un uso adecuado del poder a partir de los niveles de jerarquía establecidos y los procesos de diálogos asertivos que se establezcan entre los implicados, tomando como ejemplo el uso consecuente y racional del $1 \%$ asignado al territorio para dar soluciones concretas a las necesidades sentidas de la comunidad, donde las prácticas comunicativas juegan un papel clave en los resultados esperados.

\section{Desarrollo}

En la concepción de la innovación asumida por los municipios cubanos el hecho de ser innovador, en la actualidad, no puede ser considerado una opción entre varias, si se aspira a la competitividad, concepto que ha de ser asumido con seriedad por las municipalidades, desde la perspectiva de crear capacidades para transitar de las ventajas comparativas estáticas de un territorio, a las ventajas competitivas dinámicas, donde la tecnología, el desarrollo de procesos de aprendizaje y el incremento de la capacidad innovadora son determinantes. Núñez Jover (2013)

Para el autor de este estudio lo tributado por estos autores se materializa en la visión precisa, que tengan los gobiernos municipales en transversalizar desde su estructura, a la comunicación, como un proceso que dinamiza y operacionaliza orgánicamente estas dinámicas, desde la innovación para el desarrollo donde la voluntad política consciente al respecto, es un elemento clave de apoyatura para el proceso, ya que los decisores son fundamentales y definitorios en el desenvolvimiento de la estrategia interna asumida.

En el caso del gobierno local, la innovación en el municipio depende en gran medida de cómo sea percibida por los decisores, pues serán los gobiernos como generadores y ejecutores de políticas públicas quienes deben coordinar y controlar los trabajos para el diseño del Sistema de Innovación Local (SIL), así como lograr su implementación coherente como parte de la Estrategia de Desarrollo Municipal (EDM).

Los SIL cobran vida siempre que se logre que el gobierno los asuma como parte de una línea estratégica que reconozca a la innovación como proceso indispensable para impulsar la implantación de la EDM. Así lo muestra la Figura 1.

Figura 1. Agentes locales principales en la conexión universidad-municipio para un SIL. 


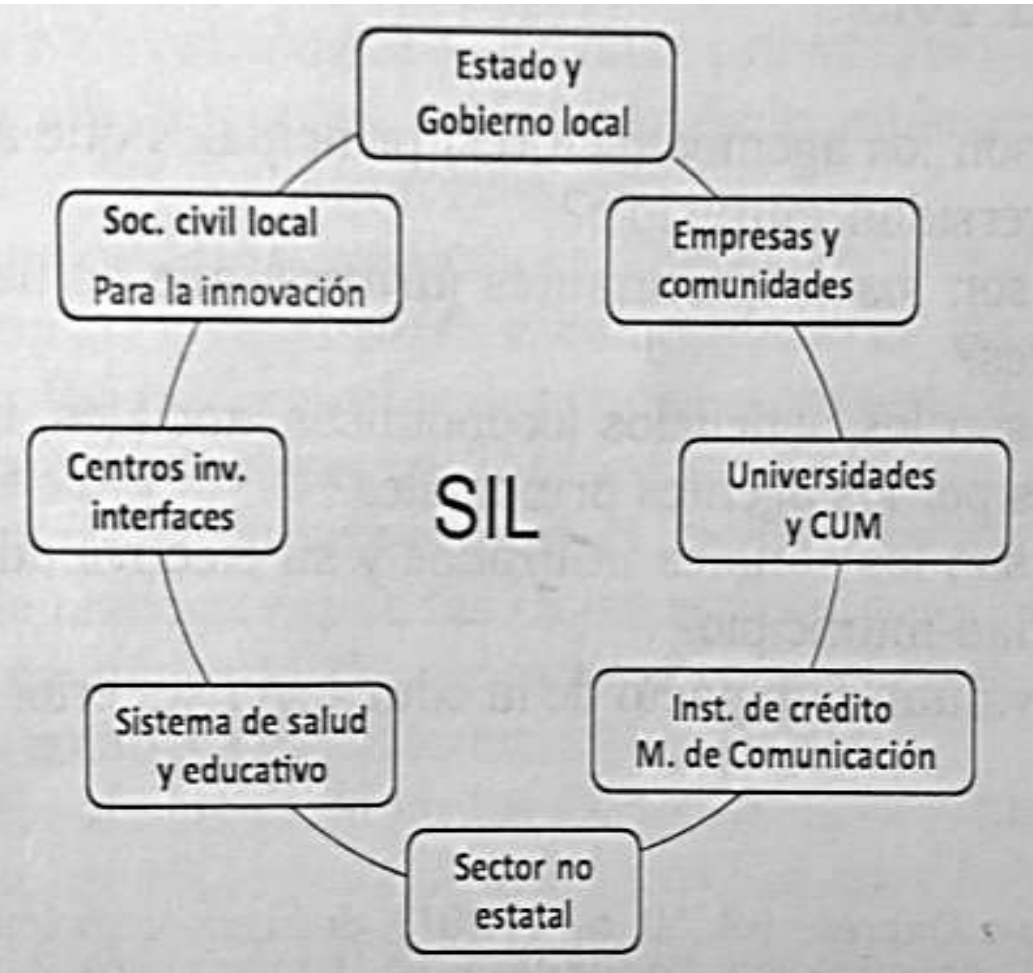

Fuente: Núñez (2018)

En cuanto a las políticas de comunicación de los gobiernos locales, las mismas deben cumplir tres funciones: la informativa, la educativa y la política. Es preciso entender que la comunicación es un proceso que aporta a la gestión del gobierno local desde las siguientes dimensiones: la institucional, aquí está presente la comunicación interna y las proyecciones hacia fuera, con sus jornadas internas, formas y espacios de recreación, sistemas de información e informáticas, los círculos de calidad y la capacitación del personal en relaciones humanas.

Otra dimensión es la discusión pública del desarrollo con la creación de espacios públicos y educativos, módulos de multimedia, estrategia de relación con medios masivos y locales. Y por último las dimensiones de participación ciudadana y la imagen y posicionamiento de la gestión y la institución municipal.

En la institucional es necesario reconocer, en que procesos y funciones se inserta la comunicación, fomentando la participación en sus diferentes áreas de gestión como: en la planificación y ejecución de proyectos de desarrollo, en la prestación de servicios públicos, en la gestión económica y financiera, en la construcción y mantenimiento de obras, en la promoción de la cultura y la identidad local, en el uso del espacio urbano y las normas de convivencia.

En la discusión pública el plan de comunicación debe garantizar gradualmente lo siguiente: las transformaciones en las personas, al interior de la municipalidad y en el municipio, todo 
esto incluyendo sus cuatro etapas bien definidas con sus componentes. En la participación ciudadana y la imagen y posicionamiento de la gestión y la institución municipal la oficina de comunicación tiene que reformular sus funciones y tener un rol más protagónico, cambiando inclusive su ubicación en la estructura municipal debiendo estar más cerca de las autoridades, de los funcionarios, de los vecinos o sea de los actores sociales.

En todo este engranaje las prácticas comunicativas, imponen un gran reto, ya que serán de hecho el elemento clave que permitirá el desenvolvimiento armónico de esta sinergia y facilitará el autodesarrollo comunitario como vía esencial para la participación, cooperación e implicación de los diferentes actores implicados. Para eso es fundamental:

1. Erradicar las prácticas comunicativas estructuradas desde posiciones transmisoras y verticalistas, provocando un cambio radical de manera que se garanticen la horizontalidad y bidireccionalidad propias de los procesos de participación democrática que la sociedad cubana reclama.

2. Potenciar la participación de los ciudadanos en los proyectos de desarrollo local constituye aún un reto para la Administración Pública.

La vinculación de la población local a los procesos de transformación desde etapas del diseño de las políticas y programas de desarrollo hasta su puesta en práctica y evaluación, necesita ser fortalecida. Como objetivo principal se acota la necesidad de abordar el "deber ser" de la Administración Pública y la utilización de la comunicación en toda su sinergia, como proceso generador de participación para asegurar legitimidad y democracia en el nuevo contexto de perfeccionamiento del modelo económico social.

\section{Prácticas comunicativas de los actores sociales}

Se presentan las prácticas comunicativas como una manera de reflejar una postura frente a los medios ciudadanos, una cualidad de actuar y apropiarse de lo que le ofrece la comunicación, y con ello, evidenciar una perspectiva política que bien puede inscribirse dentro de alguno de los paradigmas analizados por Gumucio (2004) y Vega (2011), en lo que se refiere a la relación entre la comunicación y los procesos de desarrollo.

Estos autores mencionan tres paradigmas, a saber, el paradigma dominante, en el que la comunicación juega un papel difusionista y vertical de los mensajes en pro de la modernización y la economía; o sea se entiende el papel de la comunicación en la difusión de información "de arriba hacia abajo", suponiendo que los emisores (países, especialistas, sujetos) son los desarrollados o expertos, quienes utilizan los medios como herramientas de transmisión de información y por consiguiente del ideal del desarrollo. El paradigma de la dependencia, que se presenta como respuesta a las críticas del anterior, en él la comunicación es horizontal y rescata los valores sociales y culturales de las comunidades; o sea se entiende a la comunicación como instrumento de dominación económica y cultural, viendo a los 
medios como escenarios de concentración de poder desde los cuáles se transmiten ideologías y valores, constituyéndose en instrumentos de dominación cultural.

Y el paradigma alternativo, en el que la comunicación se asume como un proceso participativo para el cambio social, o sea, se concibe a la comunicación como un proceso horizontal, de doble vía y que debe contribuir al empoderamiento de los sujetos que participan en los procesos de comunicación y de esta manera posibilitar que cada comunidad o sociedad construya su propia perspectiva del desarrollo. Vega (2011)

El concepto de prácticas comunicativas en este estudio se toma de los aportes realizados por Vega (2015), a partir de varios estudios al respecto y en los que aborda las prácticas comunicativas, en principio como elementos que reproducen identidades políticas, tomando como referente de dichas identidades a Castells (1999). Luego se asumen las identidades desde el concepto de De Certeau (1994), quien anota que en la práctica se producen significaciones, por lo que las prácticas comunicativas se conciben como prácticas culturales, especificadas en tres clases que son las legitimadoras, de resistencia y proyecto.

Finalmente, Vega (2015), aclara que las prácticas comunicativas representan, desde los conceptos de Bourdieu (1982), lógicas en la disputa por el poder al interior de los campos, en los que los agentes, a partir de ciertas posiciones y hábitos, luchan por la obtención del capital, es decir, actúan desde un rol que les es otorgado según el nivel de poder que tienen en el entorno al que pertenecen, este poder está determinado por las "reglas de juego" al interior de cada campo.

Adicionalmente, las prácticas comunicativas dan cuenta de la actuación de cada sujeto en su contexto particular, que tienen que ver de paso con elementos culturales y sociales que han sido establecidos previamente y que muestran parte de la memoria histórica a la que el agente corresponde. Respecto al tema de la relación entre prácticas comunicativas y movimientos sociales de transformación Barbero (2012) dice:

Mi propuesta es que lo que estamos necesitando es una historia de los procesos culturales en cuanto articuladores de las prácticas comunicativas con los movimientos sociales, que es, a mi modo de ver, como lo popular se inscribe constitutivamente en el análisis de los procesos históricos (p. 82)

Asumiendo a su vez la fundamentación del profesor Martín-Barbero, se retoma el concepto de práctica cultural, entendida por De Certeau (1994), como un conjunto más o menos coherente, y fluido, de elementos cotidianos concretos o ideológicos puestos al día mediante diferentes comportamientos que visibilizan socialmente fragmentos de una cultura. Prácticas que se encuentran dispersas en las acciones de un grupo social o de una sociedad, de tal forma que quienes las ejecutan, en gran medida no son conscientes de su carácter sistemático ni hereditario. 
En este sentido, los procesos comunicativos que desarrollan los diferentes actores constituyen prácticas que encarnan rituales, rutinas, procedimientos, actividades, las cuales, más que procesos simplemente técnicos se constituyen en un conjunto de elementos que configuran direccionalidades, relaciones de poder, etc., que de manera simbólica llevan implícitas posiciones ideológicas. A este tipo de significaciones o referentes simbólicos implícitos en conjunción con los procesos de "hacer la comunicación" que los incorporan es a lo que llamamos prácticas comunicativas. Pérez y Vega (2010b).

Como se puntualiza aquí, las prácticas comunicativas son elementos indispensables a la hora de revisar los procesos culturales para estudios sociales e históricos en la transformación social. Además, cada vez se hace más pertinente su abordaje, ya que, con los avances y cambios a nivel mundial, la comunicación adquiere nuevos matices y con ella las demás prácticas sociales.

Pérez y Vega (2010) han contribuido al tema en mención, desde la relación de la comunicación con el medio ambiente, ellos se centraron en las relaciones de los agentes con algún tipo de interés desde el proyecto "Identificación y caracterización de las prácticas comunicativas de los actores con intereses en el PNN Katíos" elaborado conjuntamente entre la Universidad de Antioquia y la Universidad del Norte. (Pérez y Vega, 2010b; Pérez y Vega, 2010c), clasificándolas como prácticas comunicativas legitimadoras, de resistencia o proyecto y la forma como se expresan en las esferas públicas en las cuales interactúan.

Vista las prácticas comunicativas desde este estudio el autor de la misma acota la importancia que registra como variable fundamental en el proceso histórico, cultural y social de los contextos seleccionados, donde la comunicación adquiere nuevos matices, y con ella las demás prácticas sociales.

Esto genera cambios en las relaciones de poder que se establecen entre los actores sociales, la identidad de sus rituales, rutinas, procedimientos y actividades que consolidan el hecho de cómo hacer mejor comunicación para una transformación social cualitativa y un proceso de articulación de actores a través de un dialogo asertivo, que permita una correspondencia reciproca de mensajes bien elaborados acompañados de una escucha activa para procesar información y comunicar mejor a partir de un discurso aterrizado, loable y asertivo en todo el sentido de la palabra.

Sobre la base de la definición de Vega (2014) el autor de este estudio concibe la variable como un:Proceso participativo para el cambio social, horizontal, de doble vía, que contribuye al empoderamiento de los sujetos que participan en los procesos de comunicación para posibilitar que cada comunidad construya su propia perspectiva del desarrollo.

Concluyendo podemos definir prácticas comunicativas como aquellas prácticas sociales en las que intervienen al menos dos actores sociales con funciones comunicativas diferenciadas 
de acuerdo a las circunstancias en que se desarrollen y que generalmente reproducen las regularidades de sus condiciones de existencia.

Como todo acto comunicativo, suponen la existencia de actores de la comunicación con funciones comunicativas diferenciadas de acuerdo a las circunstancias en que se desarrollen ${ }^{2}$. A saber, un productor de comunicación (EGO) y un consumidor(es) de ésta (ALTER). La realidad externa al proceso comunicativo. Las instituciones sociales condicionan la posibilidad de ser o no ser Actor en cada forma de comunicación, según la clase de referencia objeto de la comunicación. ${ }^{3}$ (Serrano, 1982: 65-81)

Concluyendo el autor de este estudio define desde la conceptualización de Vega y otros autores: a las prácticas comunicativas como aquellas prácticas sociales y culturales que constituyen un proceso participativo interactivo reflexivo de cambio social y empoderamiento, traducido en un diálogo asertivo y una articulación entre los actores sociales implicados en la búsqueda de soluciones a las problemáticas reflejadas como necesidades sentidas y reconocidas en su labor comunitaria, respaldada por un conocimiento suficiente desde sus procesos o políticas de comunicación para el desarrollo, a través de un modelo dialógico de comunicación, que permita consensos y toma de decisiones consecuentes para el beneficio social que es el objetivo principal de todos.

\section{Diálogo asertivo}

A partir del análisis del estado del arte del objeto de estudio de esta investigación, se evidencia la centralidad de la comunicación y del diálogo entre los participantes, para llevar a cabo exitosamente el desarrollo local. De ello dan fe autores tales como: Valdizán, (2007); Dollfus (1997); Corvalan y Ferreira (2003);Prieto Castillo (2000); Naciones Unidas (1997, 2006);Uranga, (2007);Arellano, (1998);Andino (2014);Rebellato. (1992,2000).Saladrigas

\footnotetext{
${ }^{2}$ Serrano define como Actores de la Comunicación a:
}

- Personas físicas que en nombre propio o como portavoces o representantes de otras personas, grupos instituciones u organizaciones entran en comunicación con otros actores.

- Las personas físicas por cuya mediación técnica unos Actores pueden comunicar con otros, siempre que su intervención técnica en el proceso comunicativo excluya, incluya o modifique a los datos de referencia proporcionados por los otros actores(Serrano, 1982: 162).

${ }^{3}$ La condición de actor viene referida, según Serrano, a la situación de estar directamente implicado en la producción, el consumo o la distribución de comunicación. En consecuencia, desde el punto de vista funcional, cabe distinguir entre dos clases de actores:

- Actores que se sirven de la comunicación. Aquellos que son responsables de la información que circula en el sistema de comunicación o aquellos que son responsables de su consumo

- Actores que sirven a la comunicación. Aquellos que ponen en circulación información elaborada por otros actores y consumida por terceros, siempre que su intervención afecte a los datos de referencia que le llegan a Alter.(Serrano, 1982: 163) 
Medina, (S/A);Portal (2000);González López. (2005);Centro de Estudios de Administración Pública (CEAP), la Facultad de Comunicación (FCOM)

Según el Dr. José A. García Higuera, la asertividad se define como: "la habilidad de expresar nuestros deseos de una manera amable, franca, abierta, directa y adecuada, logrando decir lo que queremos sin atentar contra los demás, negociando con ellos su cumplimiento". (pág.1). Está en el tercer vértice de un triángulo en el que los otros dos son la pasividad y la agresividad. Situados en el vértice de la pasividad se evita decir o pedir lo que se quiere, en la agresividad se hace de forma tan violenta que resulta inadecuada. Emplear la asertividad es saber pedir, saber negarse, negociar y ser flexible para poder conseguir lo que se quiere, respetando los derechos del otro y expresando los sentimientos de forma clara. La asertividad consiste también en hacer y recibir cumplidos, y en hacer y aceptar quejas. Higuera (2013)

Para poder ejercitar la asertividad es necesario tener capacidad de negociación, en la negociación se intenta conseguir lo que se quiere con el beneplácito del otro, que lógicamente también va a tener algunos beneficios. Higuera (2013). La realización de una negociación comprende varias fases. La primera, es la de preparación. En ella hay que pensar la estrategia seguir en el encuentro con el otro, en el que se va a plantear los problemas y se va a hacer la negociación propiamente dicha, para eso es preciso tener muy claros los objetivosque se persiguen en el encuentro, esto es lo que motiva la negociación, huir de juicios e intenciones, crear la oportunidad de negociación y recordar que siempre hay una segunda oportunidad. Higuera (2013)

\section{Articulación de actores para el desarrollo local. Requerimientos}

Articulación proviene, del vocablo latino articulatio, es el acto y el resultado de articular: la unión o la combinación de distintos elementos que otorga una cierta libertad a cada uno. Una articulación, en este marco, puede ser el vínculo que se establece entre dos piezas y que posibilita el movimiento de ambas. Los autores que la trabajan son: Alburquerque (2004),Cravacuore (2004),Madoery (2001), Barquero (2000),Ilari (2001, 2002, 2004), Cotorruelo (1995), Arocena (1998), Villar (2003, 2004, 2006, 2007), Caccia-Bava (2003), Boiser (2001), Marsiglia (2010), Caminotti; Frederic (2003), Chaqui (2015).

En los actores sociales significa un mismo pensamiento, una misma meta, dirección u objetivo a alcanzar, es lógico que existan diferencias de criterios, puntos de vista, percepciones, a partir de las vivencias y el acervo cultural de cada actor implicado, pero si estas diferencias no implican lagunas de articulación se posibilitará un movimiento uniforme hacia los objetivos que se pretenden lograr desde los cambios y transformaciones sociales con la articulación efectiva de los implicados.

En el desarrollo comunitario se precisa la articulación entre el conocimiento, el poder y la comunicación, es decir, el desarrollo como conocimiento científico y práctico, así como el 
poder que se ejerce por los actores sociales y las estrategias de comunicación que se realizan alrededor del desarrollo de la comunidad. Para el conocimiento científico el aspecto de desarrollo es un elemento que concierne en la aplicación práctica, por ello es importante, analizar como incursiona el concepto, y visualizar la relación y el comportamiento en correspondencia a las connotaciones del proceso de construcción teórica.

En esta mirada, se denota asimetría, particularmente lo que corresponde al escenario en que se establece la discusión, porque produce distanciamiento de la realidad práctica que precisa la comunidad, de tal manera que la diferencia de su concepción y aplicación es la que permite revisar el funcionamiento de la existencia de desarrollo en la comunidad. Andino (2014).

Por otra parte, es sustancial relacionar el poder, es decir, la autoridad que se ejerce desde los actores sociales en relación a la construcción de desarrollo, en este espacio participan intereses combinados; internos y externos con la intención de obtener beneficio para la comunidad, sin embargo, existen resultados poco alentadores en relación a la sostenibilidad de los proyectos de desarrollo.

Para el autor de este estudio su concepto de articulación de actores para el desarrollo se refiere a la capacidad de cada uno de los implicados de discernir y entender, que solo logrando la disponibilidad y el compromiso de trabajar todos juntos por un mismo objetivo, comprendiendo que ninguno puede por sí mismo generar el desarrollo local deseado, y que, por tanto, es imprescindible que todos conversen en conjunto para que encuentren la forma de todos juntos caminar hacia un mismo objetivo, es posible lograr el desarrollo local.

Se utilizaron los siguientes: Métodos de investigación, Técnicas e Instrumentos:

\section{Teóricos}

- Histórico-lógico: Lo histórico se refiere al estudio del objeto en su trayectoria real a través de su historia. Lo lógico interpreta lo histórico e infiere conclusiones. De la Uz, Lemus, Valdés y Padrón (2010). Se aprecia en la descripción de las dificultades de desarrollo local en los municipios y su vínculo con la Educación Superior.

- Analítico-Sintético: El análisis es un procedimiento lógico que posibilita descomponer mentalmente un todo en sus partes y la síntesis es la operación inversa, que establece mentalmente la unión o combinación de las partes previamente analizadas Martínez (2016). Se aprecia en el empleo de la elaboración de los fundamentos teóricos y metodológicos en que se sustenta el estudio, así como en el procesamiento de la información y para la interpretación de los resultados.

- Inductivo- deductivo: La inducción es una forma de razonamiento en la que se pasa del conocimiento de casos particulares a un conocimiento más general y la deducción se pasa de un conocimiento general a otro de menor nivel de generalidad Dávila (2006). Se aprecia en la determinación de los postulados generales que permiten la formulación del 
problema, y la caracterización del desarrollo local en los municipios y su vínculo con la Educación Superior.

\section{Empíricos}

- Análisis documental. Entre los métodos utilizados en el presente estudio, se analizaron las investigaciones realizadas por el Centro de Estudios de Administración Pública (CEAP), la Facultad de Comunicación (FCOM) de la Universidad de La Habana y talleres realizados con cuadros y funcionarios de la Administración Pública, en las cuales se profundizó en los procesos comunicacionales y participativos a fin de potenciar la participación de los ciudadanos en el desarrollo local.

Como resultados se detectaron muchas carencias de normas jurídicas para el trabajo con la información relevante en la Administración Local y la necesidad de potenciar los mecanismos participativos existentes a nivel local. Como conclusiones se determinó que los documentos que contienen la política de comunicación de los gobiernos locales cubanos requieren ser revisados para que se avengan a las nuevas demandas de participación ciudadana en el ámbito local.

Los Lineamientos de la Política Económica y Social del Partido y La Revolución (Partido Comunista de Cuba, 2011) liderado por su Primer Secretario y Presidente del Consejo de Ministros Raúl Castro Ruz plantean entre sus principales bases promover el desarrollo local como parte del proceso de actualización del modelo económico social, advirtiendo el tránsito hacia patrones más descentralizados de gestión, donde la administración pública asume el protagonismo a nivel local en el desarrollo económico social. PCC (2011).

\section{Conclusiones}

- Como primera conclusión de este estudio se acota que en la nueva propuesta la administración pública local, se convierte en eje decisor de la auto sustentabilidad económico financiera y transita hacia la descentralización de funciones estatales, todo ello constituye un sustancial cambio en la gestión tradicional caracterizada por la centralización por parte del Estado. En este escenario sería oportuno valorar el aporte de las estrategias comunicacionales de los gobiernos locales, a fin de potenciar la participación de la comunidad, el ciudadano y su empoderamiento en el desarrollo local, donde en este estudio desde la óptica holística ocupa un papel clave en su ejecución.

- Como segunda conclusión de este estudio partimos de que las prácticas comunicativas constituyen prácticas sociales y por tanto, se trata de conductas regulares que como tendencia no son interiorizadas a nivel del discurso aunque no se excluye, en algunos casos, esta posibilidad por efecto de la educación formal y de la inculcación sistemática a través de diversos mecanismos institucionales. 
Generalmente, reproducen las regularidades de las condiciones de existencia. Estas prácticas se desarrollan en un determinado contexto sociocultural, histórico, territorialmente determinado, por lo que están sujetas a una serie de mediaciones que dejan en mayor o menor medida su impronta en la forma en que se desarrollan, el alcance que puedan tener, pero también en sus posibilidades de modificación ante cambios en el contexto que signifiquen la apertura de posibilidades diferentes.

- Como tercera conclusión de este estudio se valora a la comunicación asertiva como una herramienta de la comunicación que favorece la comunicación eficaz entre interlocutores. Al ponerla en práctica estamos fomentando el respeto por uno mismo y respetamos a los demás. Como última conclusión de este estudio está la idea de articulación de actores. Para esto, es muy útil, intercambiar, debatir, priorizar, llegar a acuerdos y finalmente, actuar. Es decir, es necesario que estos actores se encuentren en torno a objetivos comunes de desarrollo, a través de un dialogo asertivo y prácticas comunicativas asequibles para el propósito de un beneficio en común.

\section{Referencia bibliográfica}

Alburquerque, F. (2004). "El enfoque del desarrollo económico Local". En: Cuaderno de capacitación No.1.Serie: Desarrollo Económico Local y Empleabilidad. También disponible en formato HTML: http://www.oit.org.ar/documentos/area_enfoque_del.pdf.

Alburquerque, F. (2001): "Desarrollo local en Europa y América latina"; en Vázquez Barquero, A; Madoery, O (EDS.)Transformaciones globales, instituciones y políticas de desarrollo local. Homo Sapiens. Rosario (Argentina)

Arocena, J (1998): Propuesta metodológica para el estudio de procesos de desarrollo local; Centro Latinoamericano de Economía Humana (CLAEH). Montevideo (Uruguay).

Arellano, E. (1998). La estrategia de comunicación como un principio de integración/ interacción dentro de las organizaciones. Razón y palabra, enero- marzo, Vol. 3, [Edición especial]. Extraído el 06 abril de 2009 desde http://www.Razonypalabra.org.mx/ anteriores/supesp/ estrategia.htm.

Barbero, M. (2012). Perder el “objeto" para ganar el proceso. Signo y pensamiento, 30(60), 76-84. Colombia.

Boiser, S (2001): “Desarrollo (local) ¿De qué estamos hablando?” En Vázquez Barquero, A; Madoery, O (EDS.) Transformacionesglobales, instituciones y políticas de desarrollo local. Homo Sapiens. Rosario (Argentina).

Bourdieu, P. (1982). Lección sobre la lección. Lección inaugural de la Cátedra de Sociología dictada en el Collège de France el viernes 23 de abril de 1982. 
Caccia Bava, S (2003): "Participación, representación y nuevas formas de diálogo público" En: Pobreza Urbana \& Desarrollo, IIED - América Latina, Año 9, N²1, Buenos Aires (Argentina).27-40.

Caminotti, M; Frederic. S (2003): "Hacia la generación de políticas de desarrollo local en el partido de Florencio Varela. Visiones, demandas y expectativas de los empresarios" Universidad Nacional de Quilmes. Bernal (Argentina).

Castells, M. (1999). La Era de la Información. En: El Poder de la Identidad. Madrid: Siglo XXI.

Cotorruelo M; Vázquez Barquero, A (1995): "Notas sobre la estrategia de Desarrollo Territorial"; Cuadernos de Aguilar N ${ }^{\circ}$ 5. Instituto Nacional de Empleo - Fundación de la Universidad Autónoma de Madrid. Madrid (España)

Corvalan y Ferreira (2003). Desarrollo local. Una metodología para la participación. Santiago de Chile, LOM Ediciones.

Cravacuore, D.; Ilari, S., y Villar, A.; "La articulación en la gestión local” En Cravacuore, D.; Ilari, S., y Villar, A. (2004). La articulación en la gestión municipal. Actores y políticas. Ed. Universidad Nacional de Quilmes, Buenos Aires. (Argentina). Cap. 2. $33-44$.

Chaqui (2015) "La articulación público-privada como herramienta para la innovación agrobiotecnología. El caso del Instituto de Agrobiotecnología de Rosario (INDEAR) ,2003-2015’Tesis de Licenciatura en Ciencia PolíticaOrientación en Administración y Planificación Pública.

De Certeau, M. (1994). La invención de lo cotidiano, Vol. 2. Habitar. Cocinar, México:Universidad Iberoamericana

Dollfus, O. (1997). La mundialización. París: Presses da Sciences Post

González, L. (2005).Comunicación interpersonal y comunicación organizacional: La comunicación, las relaciones humanas, y el tiempo organizacional. Colombia. Disponible en: http: //repositorio. ute.edu.ec/bistream/123456789/15718/1/459371.pdf

Gumucio-Dagron, A. (2004). "El cuarto mosquetero: La comunicación para el cambio social". Investigación y desarrollo, Universidad del Norte, 12(1), 1-22.

Herrera, E. y Vega, J. (2014). Prácticas comunicativas de participación cultural y memoria biocultural. En: A. Cadavid y A. Gumucio-Dragon (eds.), Pensar desde la Experiencia. Comunicación Participativa en el Cambio Social. Bogotá: Corporación Universitaria Minuto de Dios.

Higuera. G.J. (2013) Terapia Psicológica en el Tartamudeo, de Van Riper a la Terapia de Aceptación y Compromiso de Editorial Ariel Psicología.

Ilari, S. (2001). "La gestión local de la política social. Los casos de Quilmes y Florencio Varela”. En: Zicardi, A. (Comp.), Pobreza, desigualdad social y ciudadanía. Los límites de las políticas sociales en América Latina. CLACSO, México. 
Ilari, S. (2002a). "Estrategias de Articulación en el Marco de la Crisis en Argentina". En:Bomtempo, B.; De Carvalho, D.; De Souza, N., y Demo, P. (Orgs.), Novos Paradigmas daPolítica Social. Programa de Pos graduación en Política Social, Universidad e de Brasilia, CDU 32:33, Colección Política Social, No 1, Noviembre, Brasilia DF.

Ilari, S. (2002b). "Fragmentación y articulaciones de la política social local”. En: Cravacuore, L. (Comp.), Innovación en la Gestión Municipal. Universidad Nacional de Quilmes / Federación Argentina de Municipios, Buenos Aires.

Madoery, O (2001): "El Proyecto Político Local como alternativa de desarrollo"; Revista Política de Gestión N², Homo Sapiens. Rosario (Argentina). - Barquero, A (2000): "Desarrollo Económico Local y Descentralización: Aproximación a un marco conceptual"; En: Proyecto. CEPAL/GTZ Desarrollo Económico Local y Descentralización en América Latina. Santiago (Chile).

Marsiglia. J. (2010). ¿Cómo gestionar las diferencias?: la articulación de actores para el desarrollo local.pdf.

Núñez, Jover, J.et al. (2013) Educación Superior, innovación y desarrollo local, Revista Universidad de La Habana, n. 276. 137-162.

Pérez, M. y Vega, J. (2010a). Memorias de organizaciones juveniles, comunicación eIdentidades políticas. Estudio de caso del Colectivo Pasolini en Medellín. En: Pensar la Comunicación II (pp. 148 - 169). Medellín: Universidad de Medellín.

.. (2010b). Identificación y caracterización de las prácticas comunicativas de los actores con intereses en el PNN Katíos. Proyecto de Investigación. Universidad de Antioquia - Universidad del Norte. Documento sin publicar.

. (2010c). Aproximaciones teóricas para pensar los conflictos ambientales en el PNN Katíos: una reflexión desde la Comunicación para el Cambio Social. Conferencia ALAIC, Bogotá.

Partido Comunista de Cuba (PCC) (2011): Lineamientos de Política Económica y Social de Partido y la Revolución, Resolución VI Congreso PCC, en http: //www.cubadebate.cu.

Portal M. R 2000 La Planeación estratégica en comunicación educativa. Universidad de la Habana, Facultad de comunicación.

Prieto Castillo, D. (2000), Comunicación, Universidad y Desarrollo. Investigaciones de laPLANGESCO, La Plata.

Rebellato, J (1992). "Poder, participación popular y construcción de nuevos paradigmas". Multiversidad, no. 2. 25-47.

Rebellato, J (2000). La Participación como territorio de contradicciones éticas. En su: Antología Mínima .pág. 97-101. La Habana: Caminos.

Saladrigas Medina, Hilda: (S/A). Investigación y estrategias. Relación de concomitancia en la comunicación persuasiva. Facultad de Comunicación. Universidad de La Habana 
Serrano, M y otros. (1982). Teoría de la Comunicación. Epistemología y análisis de la referencia. Madrid, Editorial A Corazón.

Uranga, W. (2007), Mirar desde la Comunicación: Una manera de analizar las prácticas sociales. <http://www.wuranga.com.ar/images/pdfs/mira_2007.pdf>.

Valdizán García, Mª I. (2007). Universidad de Burgos y desarrollo local. Aproximación a un modelo teórico centrado en el desarrollo local desde la Universidad. (Tesis doctoral no publicada). Universidad de Burgos. Facultad de Humanidades y Ciencias de la Educación. De. De las Ciencias de la Educación.

Vázquez Barquero, A. y Madoery, O. (2001). Transformaciones Globales, Instituciones y Políticas de Desarrollo Local. Ed. Homo Sapiens, Rosario

Vega, J. (2011). Tecnologías de la información y la comunicación, subjetividad y cambio social. En: J. Pereira y A. Cadavid (eds.), Comunicación, desarrollo y cambio social. Interrelaciones entre comunicación, movimientos ciudadanos y medios (pp.349 360). Bogotá: Editorial Javeriana.

Vega, J., Pérez, M., Arango, G. y Pérez, C. (2011). Pasolini en Medellín: jóvenes, transferencia de medios y esferas públicas locales. En: Khronli, Tufte y Vega (2011) Trazos de una otra comunicación en América Latina: prácticas comunitarias, teorías y Demandas sociales (pp. 283 - 300). Barranquilla: Ediciones Uninorte.

Vega, J. (2015). Prácticas comunicativas, habitus e identidades políticas en procesos de comunicación local. Libros Editorial UNIMAR. Colombia

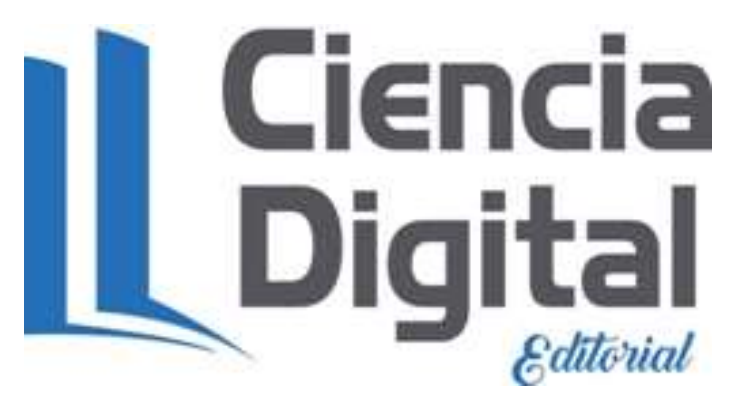


Para citar el artículo indexado.

Santa Cruz D, Ojalvo V., Valdés Y. \& Velasteguí E. (2019) Dificultades de desarrollo local en los municipios y su vínculo con la Educación Superior. Revista electrónica Ciencia Digital 3(2), 353-369. Recuperado desde: http://cienciadigital.org/revistacienciadigital2/index.php/CienciaDigital/article/view/355/913

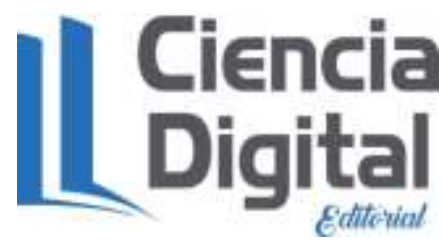

El artículo que se publica es de exclusiva responsabilidad de los autores y no necesariamente reflejan el pensamiento de la Revista Ciencia Digital.

El articulo queda en propiedad de la revista y, por tanto, su publicación parcial y/o total en otro medio tiene que ser autorizado por el director de la Revista Ciencia Digital.
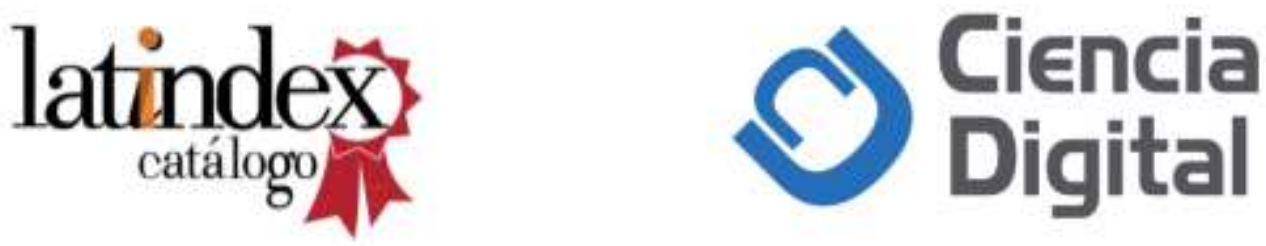